\title{
Chemodynamical Evolution of the Milky Way
}

\author{
Chiaki Kobayashi ${ }^{1}$, Naohito Nakasato ${ }^{1,2}$, and Ken'ichi Nomoto ${ }^{1}$ \\ ${ }^{1}$ Department of Astronomy, School of Science, University of Tokyo \\ 2 Astronomisches Rechen-Institut
}

\begin{abstract}
We simulate the chemodynamical evolution of the Milky Way using our GRAPE-SPH code, and reproduce the age-metallicity relation, the $[\mathrm{O} / \mathrm{Fe}]-[\mathrm{Fe} / \mathrm{H}]$ relation, and the metallicity distribution.
\end{abstract}

\section{Introduction}

The high-quality observations of galaxies with HST and 8M telescopes provide the detail information of spectro-photometric, chemical, and kinematic properties. To interpret such observations and investigate how galaxies form and evolve, it is necessary to compare the observational data with the theoretical predictions. Thus, realistic galaxy models are required.

Here we present the results of the numerical simulation of the formation and evolution of the isolated galaxy that consists of dark matter, gas, and stars. To construct the self-consistent three-dimensional chemodynamical models, we introduce the various physical processes associated with the formation of stellar systems; radiative cooling, star formation, feedback of Type II and Ia supernovae (SNe II and SNe Ia), and stellar winds (SWs), chemical enrichment, and the UV background radiation. However, there are some assumptions in such physical processes. Here we construct a model of Sb galaxy and compare the observational data obtained in the Milky Way, which can be a significant test of such assumptions.

\section{Model}

i) We adopt the Smoothed Particle Hydrodynamical (SPH) method, and the gravity is computed by the GRAPE. Our code is highly adaptive in space and time by means of individual smoothing length and individual timestep. ii) We use the metallicity-dependent cooling function. For the primordial gas, the effect of the homogeneous isotropic UV background radiation is introduced (Katz, Weinberg, \& Hernquist 1996). iii) The star formation criteria are 1) convergent, 2) cooling, and 3) Jeans unstable. We also include the probability criterion. The star formation rate is determined from the Schmidt law. iv) For the feedback of energy and heavy elements, we exclude the instantaneous recycling approximation. For SWs, SNe II, and SNe Ia, one star eject the thermal energy to the surrounding gas particles within $1 \mathrm{kpc}$. We adopt the Salpeter initial mass function. For SNe Ia, we adopt the single degenerate scenario with the metallicity effect (Kobayashi et al. 1998, 2000). v) As the initial condition, we use the CDM initial fluctuation, which is generated by the COSMICS (E. Bertschinger). We simulate the 3- $\sigma$ over dense region with the radius of $55 \mathrm{kpc}$, the mass of $\sim 10^{12} M_{\odot}$ (baryon fraction of 0.1), $\sim 40000$ particles (the half for gas and the rest for dark matter), and the spin parameter of $\lambda \sim 0.1$. 

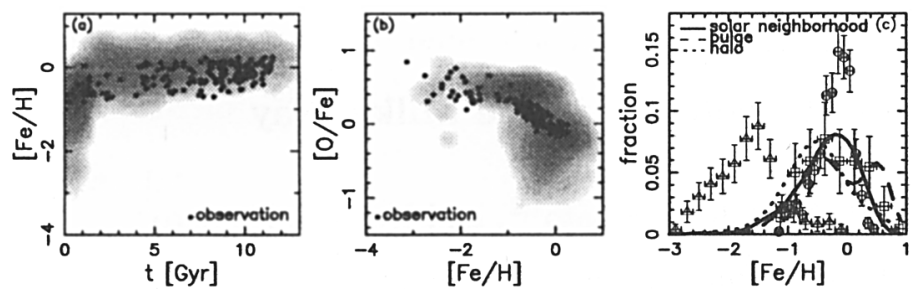

Figure 1. (a) Age-metallicity relation and (b) $[\mathrm{O} / \mathrm{Fe}]-[\mathrm{Fe} / \mathrm{H}]$ relation in the solar neighborhood. The contours show the mass density for the simulation, and the dots show the observation. (c) Metallicity distribution functions in the solar neighborhood (solid line, circles), bulge (dashed line, squares), halo (dotted line, triangles). The symbols with errorbars show the observation.

\section{Results and Discussion}

The merging of sub-galaxies induces the initial star burst and the bulge forms by $z \gtrsim 3$. The gas accrete on the disk and disk stars formed at $1 \lesssim z \lesssim 3$. The bulge of the simulated galaxy has the de Vaucouleurs surface brightness profile and the steep metallicity gradients of $\Delta[\mathrm{Fe} / \mathrm{H}] / \Delta \log r \sim-0.5$, and the disk has the exponential surface brightness and the shallow gradients of $\Delta[\mathrm{O} / \mathrm{H}] / \Delta r \sim-0.005$, which are consistent with the observation. (a) In Figure 1a, we reproduce the age-metallicity relation. $[\mathrm{Fe} / \mathrm{H}]$ increases to $\sim 0$ at $t \sim 2$ Gyr. (b) In Figure $1 \mathrm{~b}$, we reproduce the $[\mathrm{O} / \mathrm{Fe}]-[\mathrm{Fe} / \mathrm{H}]$ relation. $[\mathrm{O} / \mathrm{Fe}]$ decreases because of the delayed iron enrichment of $\mathrm{SNe}$ Ia. If we do not include the metallicity effect on $\mathrm{SNe} \mathrm{Ia}$, we cannot reproduce the plateau of $[\mathrm{O} / \mathrm{Fe}] \sim 0.45$ at $[\mathrm{Fe} / \mathrm{H}] \lesssim-1$. (c) Figure 1c shows the metallicity distribution functions. For the bulge, the simulated distribution is in good agreement with the observation. For the solar neighborhood, the peak is consistent but the number of metal poor stars is a little larger than the observation (i.e., the G-dwarf problem). Since we include the effect of the UV background radiation, the number of metal-poor stars is smaller than the case without the effect. For the halo, the peak is higher than the observation. These are because the star burst occurs at the very early epoch before gas accrete onto the disk. There may be room to improve the criteria of star formation, and the initial mass function may depend on the metallicity.

\section{Conclusions}

We construct the detail model of the formation and evolution of the Milky Way using our GRAPE-SPH code. The simulated Sb galaxy has the three components; bulge, disk, and halo, of which dynamical and chemical properties are consistent with the observation in the Milky Way. We reproduce the agemetallicity relation and the $[\mathrm{O} / \mathrm{Fe}]-[\mathrm{Fe} / \mathrm{H}]$ relation in the solar neighborhood, and mostly reproduce the metallicity distribution functions in the Milky Way.

\section{References}

Katz, N., Weinberg, D.H., \& Hernquist, L., 1996, ApJS, 105, 19

Kobayashi, C., et al. 1998, ApJ, 503, L155

Kobayashi, C., Tsujimoto, T., \& Nomoto, K. 2000, ApJ, 539, 26 